\title{
Control of mixing in a Stokes' fluid flow
}

\author{
Ian J. Couchman ${ }^{\mathrm{a}, \mathrm{b}}$, Eric C. Kerrigan ${ }^{\mathrm{a}, \mathrm{b}, *}$ \\ ${ }^{a}$ Department of Electrical and Electronic Engineering, Imperial College London, Exhibition Road, London, \\ SW7 2AZ, United Kingdom. \\ ${ }^{b}$ Department of Aeronautics, Imperial College London, Exhibition Road, London, SW7 2AZ, United Kingdom.
}

\begin{abstract}
Mixing problems are common in science and engineering, the aim being to combine fluids as quickly and efficiently as possible. We consider the design of a simple controller to promote mixing in a Stokes' fluid flow. In this paper, a controlled stirring motion is represented by a velocity field consisting of the superposition of a steady base flow and a second field modulated by a saturating, time-dependent control variable. The problem can be formulated as an optimal control one, but the presence of a nonlinearity in the state dynamics and an input constraint make the construction of a feedback law difficult. The size of the problem means that receding horizon schemes, revolving around real-time optimization of even a simplified model, are currently not feasible for fast applications. To address this problem, we exploit theory for the control of bilinear systems to propose a simple, well-performing, explicit feedback law. There are several interesting design issues associated with applying this approach to a fluid mixing application. We demonstrate these by designing a controller for a two-dimensional fluid mixing problem with simple cellular flows and discuss the relevant implementation decisions. The closed-loop forcing fields have many features that are as expected: regions of the flow with large spatial velocity gradients target regular islands of concentration and the input favors velocity fields with contours aligned perpendicular to the scalar field.
\end{abstract}

Keywords:

Bilinear systems; Fluid mixing; Large-scale systems.

\section{Introduction}

The promotion and control of mixing is highly relevant to modern and future engineering applications, e.g. in the fields of biotechnology, pharmaceutics and lab-on-a-chip processes [1]. In [2] mixing is described as the process of reducing inhomogeneity in a distribution in order to achieve a desired result. The inhomogeneity could be temperature or concentration, for example, and is represented by a scalar field. The scalar field evolves according to a stirring motion, represented by a velocity field and molecular diffusion, resulting from scalar gradients. The aim in a mixing process is to influence the stirring motion in order to create scalar gradients at the correct places and times in order for diffusion to mix the distribution most efficiently. In some applications, it is sufficient to use a steady stirring motion to mix the field, while relying on heuristics to know when

\footnotetext{
${ }^{*}$ Corresponding author

Email addresses: ian.couchman06@imperial.ac.uk (Ian J. Couchman), e.kerrigan@imperial.ac.uk (Eric C. Kerrigan)
} 
the product is suitably mixed for the desired use [2]. However, industry is driven by profit, and a lack of efficiency in the mixing process can be particularly expensive; for example, in 1993 the US chemical industry estimated its losses due to poor mixing at $\$ 100$ million [2]. In these cases, more effective solutions are necessary. Often such applications are further constrained by the need for the flow to move in a simple fashion known as laminar flow [2]. The necessity for laminar flow can be as a result of a number of factors:

- The size of the domain, e.g. in microfluidics [3];

- High viscosities, e.g. food processing [2];

- Low speeds, e.g. pharmaceutics [2];

- Shear sensitive materials, e.g. biotechnology [4].

A popular way of mixing in these laminar cases is to use time-varying stirring protocols designed to effectively stretch and fold blobs of the scalar [5]. It is possible to force and control a velocity field, see for example $[2,6,7]$, and so it is relevant to consider how to best advect an initial distribution.

Many engineering principles have been developed for the design and construction of mixing equipment for specific applications. A summary of these principles, ranging from rules-of-thumb and ad hoc approaches to schemes implemented with theoretical results in mind can be found in [2]. For example, work presented in [8,9] details a simplified model for mixing in a torus reactor and goes on to investigate the use of this model for the design of stirring conditions. For such an application, a well performing forcing function can be related to certain time properties of the flow.

In contrast, work has been published in the fluids and applied maths communities investigating the optimal velocity fields. These studies can be loosely categorized by the level at which mixing is judged. In $[10,11,12]$ the aim is to generate and control velocity fields to have certain asymptotic properties known to mix well or even to destabilize the flow. These works are not dependent upon the initial orientation of the scalar field and therefore it is reasonable to expect them to be suboptimal. To counter this, some research has focussed on the construction of velocity fields with the aim of mixing a given initial condition.

In [13] calculus of variations is used to derive optimality conditions that must be satisfied by a velocity field in order to mix an initial scalar field with respect to a mixing cost function for the purely advective and advective-diffusive cases. A drawback of the approach of [13] is that it may be difficult to physically realize the velocity field found. In [14], [15] this issue is addressed by considering velocity fields that can be written as the superposition of reasonable velocity fields. The work presented in [16] considers mixing protocols for a switched sine flow. Two interesting conclusions are made; firstly, there exist many protocols all with similar performance and secondly, a well performing mixing protocol is insensitive to plant diffusivity.

Research into optimal mixing of a given distribution has almost always focused on the computation of open-loop mixing protocols. The reason for this is that the problem has nonlinear dynamics, constraints and is infinite-dimensional in nature, a problem for which no explicit feedback solution exists. A spatial discretization of the plant leads to a model with an extremely large state vector, typically of the order of $10^{3}-10^{6}[15,16]$. The time involved in solving the open-loop optimization problems posed in $[15,16]$ currently prohibits recursive solution approaches like receding horizon control for fast applications. It is well-known in the robust control literature that open-loop solutions could be very conservative because often they want a solution that works for all initial states. A solution that is a function of the current state is known to be much less 
conservative. The computation of the optimal open-loop solution does shed light on the physics of mixing, but the presence of disturbances, for example changes in diffusivity, model-plant mismatch and the fact that in many practical applications the initial conditions may vary, do not promote them as engineering solutions. It is this computation of open-loop solutions and the analysis of their physical nature that dominates the fluids literature. In contrast, this paper focuses on the issues associated with the design of a controller to promote mixing that is easily adaptable to different geometries and applications. The nature of the contribution is in exploiting both results on the control of constrained bilinear systems and observations from the fluids literature about the lack of sensitivity of well-performing input sequences to model diffusivity [16] to design such a controller.

We consider velocity fields that are induced by the superposition of a steady base flow and a second flow modulated by a saturating, time-varying, control input. For example, think of a device where species need to be combined and there is one main circulating flow and an additional controlled stirring motion. How should we add this second controlled part to improve performance? Is the solution a viable option for real-time applications? We will see the answer to the former can be written as a simple feedback law such that the answer to the latter is 'yes'.

Recently, an indicator of the degree of mixedness of a scalar field over all scales was introduced in [17], known as the mix-variance. It has been shown to be equivalent to a Sobolev space norm of the difference between the scalar field and its mean [17]. Although other multiscale measures exist, this equivalence means that the mix-variance can be written as a quadratic function of the state [17], which is necessary to exploit the approach proposed in this paper.

The state dynamics considered in this paper are governed by a bilinear, partial differential equation (PDE), known as the advection-diffusion equation. A bilinear PDE is one that is infinitedimensional, linear in the state and linear in the input but not jointly linear in both. Physical limitations result in simple upper and lower bounds on the input. The optimal control problem can then be seen as a constrained, single-input, bilinear quadratic regulator problem. Currently no explicit solutions exist to such problems, but in [18] the quadratic cost functional is modified by the addition of a nonnegative state-penalizing term in the integrand. This 'regularized' problem admits a simple, closed-form solution. In [18] this approach is demonstrated to be only marginally sub-optimal, although currently no prior performance bounds exist.

We note at this point that the feedback solution we propose is a state-feedback solution. Typically in large-scale control applications, measuring the whole state would be impractical. However, techniques such as laser induced fluorescence [19] facilitate the proposed methodology from a practical perspective.

In Section 2, we formulate the problem mathematically. The solution approach is described in Section 3, and demonstrated on a numerical example in Section 4. The paper concludes with a summary of the advantages and disadvantages of the method from Section 3 and a proposition for future work.

\section{Problem formulation}

In this section we formulate the optimal control of mixing problem to be considered in this paper. We start by describing the dynamics of the mixing process and discussing some relevant properties in Section 2.1. In Section 2.2 we introduce the concept of what it means for a scalar field to be well mixed and use this to detail the objective by which we will judge performance. Finally, we formally define the optimal control problem that we wish to solve in this paper in Section 2.3. 


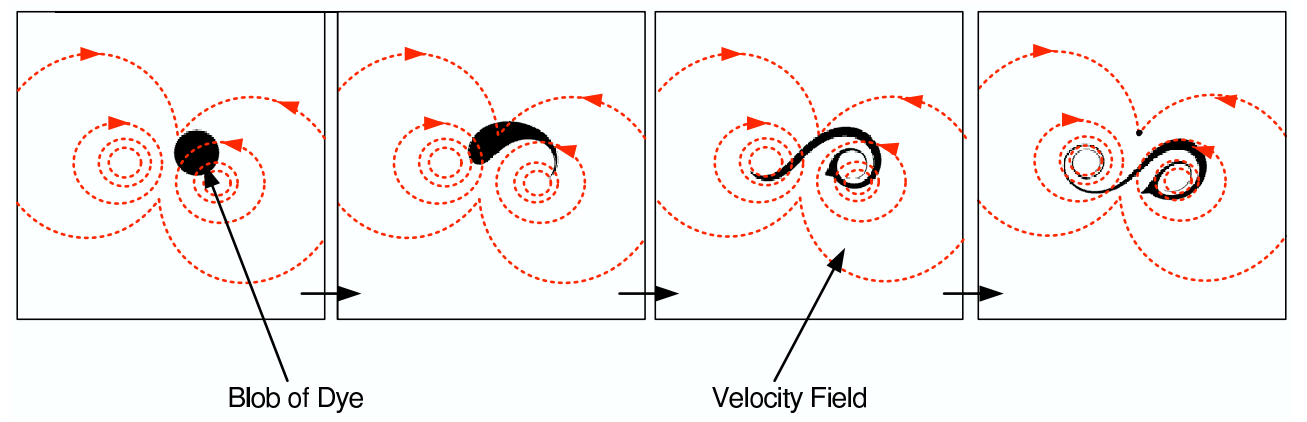

Figure 1: Blob of dye evolving under the action of a velocity field. We are looking to vary this velocity field with time to mix the blob most effectively.

\subsection{State evolution}

We study fluid mixing in a two-dimensional domain $\mathcal{D}:=[0,1] \times[0,1] \subset \mathbb{R}^{2}$ as in $[16]$ and $[15]$, equipped with periodic boundary conditions because it simplifies computation and presentation of the mathematics. The applicability of the work to more complicated geometries and boundary conditions is discussed in Section 3.1. In a flow defined over $\mathcal{D}$, a scalar field $x: \mathcal{D} \times[0, \infty) \rightarrow \mathbb{R}$, such as temperature or concentration, is advected by the action of a velocity field and diffused as a result of scalar gradients. It is possible to generate and control velocity fields by applying a force field to the system in some way. For example, in microfluidic mixing applications, magnetohydrodynamics is a viable option [7], while in industrial mixers, impellers are commonly used [2].

A Stokes' flow is a flow where advective inertial forces are small in comparison to viscous forces [20, p. 41]. This is a typical situation in microfluidic devices, for example, due to the length scales involved [3], or in polymer manufacturing [2]. A Stokes' flow has a linear mapping between the forcing function and the flow velocity field [20, p. 41]; if a pair of forcing functions $g_{1}, g_{2}: \mathcal{D} \rightarrow$ $\mathbb{R}^{2}$ establish velocity fields $f_{1}, f_{2}: \mathcal{D} \rightarrow \mathbb{R}^{2}$, respectively, then in accordance with Stokes' relation, the forcing function $g:=g_{1}+u g_{2}, u \in \mathbb{R}$ will generate the velocity field $f:=f_{1}+u f_{2}$. This simplifying assumption allows us to neglect the dynamics associated with inducing the velocity field. In the case of magnetohydrodynamics [7], $g$ would be an electromagnetic forcing field that interacts with a current passed through the fluid to generate the velocity field $f$.

To help visualize this set-up, think of the case of dropping a blob of dye into a tray of white liquid. Figure 1 shows the evolution of a black blob under the action of the velocity field shown. In this work we are looking to define a time-varying velocity field in order to distribute the blob evenly across the domain most efficiently.

The velocity field $f: \mathcal{D} \times[0, \infty) \rightarrow \mathbb{R}^{2}$ we consider comprises of the superposition of two incompressible fields $f_{1}:=\left[f_{1}^{1} f_{1}^{2}\right]^{\prime}, f_{2}:=\left[f_{2}^{1} f_{2}^{2}\right]^{\prime}$, where $f_{1}, f_{2}: \mathcal{D} \rightarrow \mathbb{R}^{2}$. The incompressibility assumption represents the fact that the fluids considered are of uniform density and implies that the velocity fields are divergence-free, i.e. $\nabla \cdot f_{1}:=\frac{\partial f_{1}^{1}}{\partial y_{1}}+\frac{\partial f_{1}^{2}}{\partial y_{2}}=0, \nabla \cdot f_{2}=0$ where $\nabla:=\left[\frac{\partial}{\partial y_{1}} \frac{\partial}{\partial y_{2}}\right]^{\prime}$ and $y:=\left[\begin{array}{ll}y_{1} & y_{2}\end{array}\right]^{\prime} \in \mathcal{D}$ is the location in space. This simplifies the dynamics because we can ignore any dynamics associated with fluctuations in fluid density. Due to the periodic boundary conditions of $\mathcal{D}$, an additional requirement on $f$ is that it also satisfies periodic boundary conditions, i.e.

$$
f\left([0 z]^{\prime}, t\right)=f\left([1 z]^{\prime}, t\right), \quad f\left([z 0]^{\prime}, t\right)=f\left([z 1]^{\prime}, t\right), \quad \forall z \in[0,1], t \in[0, \infty) .
$$

The control variable $u:[0, \infty) \rightarrow \mathbb{R}$ modulates the component of the second field, so $f$ is defined 


$$
f(y, t):=f_{1}(y)+u(t) f_{2}(y) .
$$

The advection-diffusion equation governs the behavior of a passive scalar field $x(\cdot)$ under the action of such a flow. The state evolution satisfies

$$
\begin{aligned}
\frac{\partial x}{\partial t}(y, t) & =\kappa \Delta x(y, t)-\left(f_{1}(y)+u(t) f_{2}(y)\right)^{\prime} \nabla x(y, t), \\
x(y, 0) & =x_{0}(y), \\
x\left([0 z]^{\prime}, t\right) & =x\left([1 z]^{\prime}, t\right), \quad x\left([z 0]^{\prime}, t\right)=x\left([z 1]^{\prime}, t\right), \quad \forall z \in[0,1],
\end{aligned}
$$

$\forall t \in[0, \infty), y \in \mathcal{D}$, where (2c) represents periodic boundary conditions, $\kappa>0$ is the molecular diffusivity, $\nabla x:=\left[\frac{\partial x}{\partial y_{1}} \frac{\partial x}{\partial y_{2}}\right]^{\prime}$ and $\Delta x:=\frac{\partial^{2} x}{\partial y_{1}^{2}}+\frac{\partial^{2} x}{\partial y_{2}^{2}}$. The initial concentration field $x_{0}: \mathcal{D} \rightarrow \mathbb{R}$ is a function in the space $\mathcal{L}_{\mathcal{D}}^{2}$ of all measurable functions mapping $\mathcal{D} \rightarrow \mathbb{R}$ whose square of the absolute values has a finite Lebesgue measure. All this condition says is that the initial condition is not infinite anywhere and is sufficient in order to write the state field as a Fourier series [21]. For all physical cases, this assumption is in no way restrictive. The diffusivity stays constant throughout. The input is constrained to $[-1,1]$, representative of physical limitations on the source ${ }^{1}$. It is worth noting that the Stokes' linearity assumption allows the velocity field $f_{2}$ to be scaled arbitrarily; it does not imply that the input $u(\cdot)$ to state $x(\cdot)$ behavior is linear; it is in fact a bilinear system.

To conclude this section, we discuss some properties of the state dynamics that we exploit in Section 3.1 in order to simplify the control problem. The following results are well-known in the fluids community and together they simply imply that if one adds red and white liquids, it doesn't matter how you stir it, you will eventually end up with a pink liquid. Furthermore, the shade of pink depends only upon the initial condition and once the mixture is pink, no further stirring can affect it.

Proposition 1. The solution $x(\cdot)$ to $(2)$ has the following properties for all feasible input functions $u:[0, \infty) \rightarrow[-1,1]$ :

1. The mean of the scalar field is conserved, i.e.

$$
\int_{\mathcal{D}} x(y, t) \mathrm{d} y=\bar{x}_{0}, \quad \forall t \in[0, \infty),
$$

where $\bar{x}_{0}:=\int_{\mathcal{D}} x_{0}(y) \mathrm{d} y$ is the mean of the initial condition;

2. As $t \rightarrow \infty, x(y, t) \rightarrow \bar{x}_{0}, \forall y \in \mathcal{D}$;

3. If $x_{0}(y)=\bar{x}_{0}$ for all $y \in \mathcal{D}$, then $x(y, t)=\bar{x}_{0}$ for all $y \in \mathcal{D}, t \in[0, \infty)$.

A proof of these properties can be found in [22].

\footnotetext{
${ }^{1}$ This is without loss of generality, since the general case $u(t) \in[a, b]$ can be mapped to a system with symmetric unit input constraints with a simple change of variable, $u=\frac{a+b}{2}+\frac{b-a}{2} \tilde{u}$ so $f(y, t)=\tilde{f}_{1}(y)+\tilde{u}(t) \tilde{f}_{2}(y)$ where $\tilde{f}_{1}(y):=f_{1}(y)+\frac{a+b}{2} f_{2}(y), \tilde{f}_{2}(y):=\left(\frac{b-a}{2}\right) f_{2}(y), \tilde{u}(t) \in[-1,1]$ for all $t \in[0, \infty)$.
} 


\subsection{Cost function}

A multiscale measure for mixing was presented in [17] to quantify the degree of mixedness of a scalar field. The so-called mix-variance considers the energy of the deviation of $x(\cdot, t)$ from its mean over the whole of $\mathcal{D}$, for a range of subsets of $\mathcal{D}$. The periodic boundary conditions imply that the wavelengths in the scalar field will be between 0 and 1 in both spatial directions. To incorporate the mixedness over this range of scales, the subsets considered are balls $\mathcal{B}(p, s)$ of radius $\frac{s}{2}, s \in(0,1)$ with center $p \in \mathcal{D}$ defined as

$$
\mathcal{B}(p, s):=\left\{z \in \mathcal{D} \mid\|z-p\|_{2} \leq \frac{s}{2}\right\} .
$$

Proposition 1 implies that $\int_{\mathcal{D}} x(y, t) \mathrm{d} y=\bar{x}_{0}$ for all $t \in[0, \infty)$. The set $\mathcal{B}(p, s)$ has area $\frac{1}{4} \pi s^{2}$ and therefore the mean of the difference between $x(\cdot, t)$ and its mean over $\mathcal{D}, \bar{x}_{0}$, over $\mathcal{B}(p, s) \subset \mathcal{D}$ can be written as

$$
\frac{4}{\pi s^{2}} \int_{\mathcal{B}(p, s)} x(y, t)-\bar{x}_{0} \mathrm{~d} y .
$$

Summing the square of this quantity, i.e. energy in the deviation, for all feasible $\mathcal{B}(p, s)$ yields the mix-variance $\Phi^{2}(x(\cdot, t))$, defined as

$$
\Phi^{2}(x(\cdot, t)):=\int_{0}^{1} \int_{\mathcal{D}}\left(\frac{4}{\pi s^{2}} \int_{\mathcal{B}(p, s)} x(\cdot, t)-\bar{x}_{0} \mathrm{dy}\right)^{2} \mathrm{~d} p \mathrm{~d} s .
$$

If the field $x(\cdot, t)$ is completely mixed, then the mean of $x(\cdot, t)$ over every subset of $\mathcal{D}$ will be the same as the mean of the field over $\mathcal{D}$ and so the mix-variance will be zero. This quantity has parallels with the concept of a process being 'mixing' in the ergodic theoretic sense, a discussion of which can be found in [17].

For functions in $\mathcal{L}_{\mathcal{D}}^{2}$, the mix-variance can be simply interpreted in the Fourier domain. Consider the Fourier series representation of the state field, which exists for any function $x(\cdot, t) \in \mathcal{L}_{\mathcal{D}}^{2}[21$, Sect. 2.1.1]:

$$
x(y, t)=\sum_{\substack{k_{1}, k_{2}=-\infty \\ k_{1}, k_{2} \in \mathbb{Z}}}^{\infty} \tilde{x}(k, t) \exp \left(2 \pi \mathrm{i} k^{\prime} y\right), \forall y \in \mathcal{D}, \quad \forall t \in[0, \infty),
$$

where $\tilde{x}: \mathbb{Z}^{2} \times[0, \infty) \rightarrow \mathbb{C}$ are the Fourier coefficients, the wavenumber $k:=\left[k_{1} k_{2}\right]^{\prime}$ and $\mathrm{i}:=\sqrt{-1}$. For such a function, the mix-variance is equivalent to a Sobolev space norm of negative index $-\frac{1}{2}$ of the difference between $x(\cdot, t)$ and its spatial mean $\bar{x}_{0}$, and hence can be represented as [17]

$$
\left\|x(\cdot, t)-\bar{x}_{0}\right\|_{H^{-\frac{1}{2}}}^{2}:=\sum_{k} \frac{1}{\left(1+4 \pi^{2}\|k\|_{2}^{2}\right)^{\frac{1}{2}}}|\tilde{x}(k, t)-\tilde{x}(0, t)|^{2},
$$

where $\|\cdot\|_{2}$ denotes the vector 2 -norm and $|\cdot|$ denotes the magnitude of a number in $\mathbb{C}$. The mixvariance can then be interpreted as a weighted sum of the energy in each of the Fourier coefficients. This is particularly useful in the computation of the mix-variance because efficient techniques for 


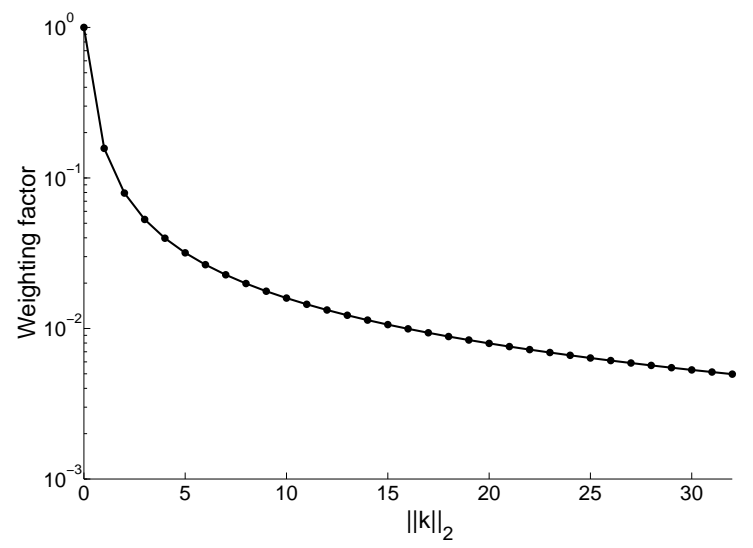

Figure 2: Log-linear plot of the weighting factor in the cost versus the norm of the wavenumber.

computing the Fourier transform exist [23]. Figure 2 shows the weighting factor versus the norm of the wavenumber. The lower the wavenumber, the more harshly it is penalized in the cost. This makes physical sense: if our tray had red liquid in one half of the domain and white in the other, all the energy would be in the lowest Fourier modes and so the composition would be seen as poorly mixed.

\subsection{The optimal control problem}

Due to the fact that the dynamics conserve the mean of the scalar field and the cost function depends only on deviation from the mean, we consider only zero mean initial conditions, i.e. $\bar{x}_{0}=0$, without loss of generality. When this is not the case, the state $x$ simply becomes the deviation of the scalar field from its mean. For a discussion of the justification of this for the more general problem of optimizing a similar cost function over all time-varying, incompressible velocity fields, as opposed to just ones that can be written in the form of (1), see [13].

The optimal control problem to be solved can then be written in standard form as

$$
\min _{u(\cdot), x(\cdot)} \int_{0}^{\infty}\|x(\cdot, t)\|_{H^{-\frac{1}{2}}}^{2}+\frac{1}{2} r u^{2}(t) \mathrm{d} t,
$$

subject to $(2)$ and

$$
-1 \leq u(t) \leq 1, \quad \forall t \in[0, \infty),
$$

where $x_{0}(\cdot)$ is the given, zero mean, initial condition and $r>0$ determines the ratio of the penalization of cost to control input energy. By using the sum of the mix-variance and the square of the input as the stage cost we aim to achieve a trade-off between how fast the fluid converges to a well-mixed field and the amount of control energy used.

\section{Solution}

In Section 2 we formulated the optimal control of mixing problem mathematically as a constrained infinite-dimensional problem. There is a significant body of theory on the control of distributed parameters systems, however the techniques described usually generate infinite dimensional controllers [24]. These are clearly not implementable in practice. In order to get finite dimensional approximations, control engineers have two options: 
1) Design an infinite dimensional controller, see for example [24], and then discretize the controller;

2) Discretize the dynamics and then design the control law.

As no solutions exist to the constrained infinite-dimensional control problem (5), we adopt the latter approach. In this section we introduce the proposed solution approach. We begin in Section 3.1 by discretizing the problem spatially to get a finite-dimensional optimal control problem and detail the model used for the simulation of the plant. We then exploit some of the properties of the dynamics introduced in Section 2.1 to get a system realization that is suitable for use in control design. In Section 3.2 we describe the method from [18] for the control of bilinear systems and explain its applicability to the mixing of fluids problem. We follow this with a discussion on the physical interpretation of the feedback law in Section 3.3. This is important because the proposed controller shows many features one would expect physically from a well-performing control law. Although there are no performance guarantees, this analysis goes some of the way to justifying the efficacy of the scheme. In Section 3.4 we discuss the sensitivity of the proposed law to certain physical properties of the plant. Combining this discussion with the observations introduced in Section 3.3 we are then in a position to introduce the relevant design decisions associated with the proposed control law.

We note that there are no assurances that the control law designed for a discretized model of the plant will stabilize the true, infinite dimensional plant. Only recently are such results beginning to appear in the control literature for linear systems [25], but currently no such results exist for nonlinear systems. In an attempt to justify our approach, Section 4 contains a detailed study of the effects of discretization on the closed-loop performance of the controller applied to a numerical example.

\subsection{Obtaining a suitable finite dimensional model}

In order to employ classical control system design methods, we need a finite dimensional state space model. To achieve this, we project the infinite-dimensional state dynamics onto a finite dimensional subspace with a Fourier-Fourier discretization [21]. We chose periodic boundary conditions to facilitate the use of such a discretization because of its simplicity, but the scheme is in no way dependent upon it. In fact, the methods described will work for any boundary condition and discretization under the very loose proviso that the discretized system and cost take the form of (6) and (7) below, respectively.

We define an equispaced grid of points across the domain $\mathcal{D}$ contained in the $n^{2}$-tuple $\mathcal{Y}_{n}:=$ $\left(v_{1}, v_{2}, \ldots, v_{n^{2}}\right)$ where each $v_{i} \in \mathcal{D}$ is defined as

$$
v_{i}:=\left[\frac{i-1-(i-1) \bmod n}{n^{2}} \frac{i \bmod n}{n}\right]^{\prime}, \quad i=1,2, \ldots, n^{2},
$$

where mod denotes the modulo division operator. Approximations $X_{i}(t) \approx x\left(v_{i}, t\right), i=1,2, \ldots, n^{2}$, are found by replacing spatial derivatives in (2) by Fourier differentiation matrices $[26,27]$ and the state by the vector of approximates $X(t):=\left[X_{1}(t) X_{2}(t) \cdots X_{n^{2}}(t)\right]^{\prime}$. We get a set of ordinary differential equations

$$
\dot{X}(t)=\left(A_{\kappa}+N_{0}\right) X(t)+u(t) N X(t), \quad X(0)=\left[x\left(v_{1}, 0\right) \cdots x\left(v_{n^{2}}, 0\right)\right]^{\prime},
$$

where $A_{\kappa}, N_{0}, N \in \mathbb{R}^{n^{2} \times n^{2}}$ are defined in Appendix A. We use separate symbols for $A_{\kappa}$ and $N_{0}$ to explicitly separate the advective and diffusive components. This makes the discussion that follows 
into the effects of diffusivity clearer. We use $\left(A_{\kappa}, N_{0}, N\right)$ to denote the realization (6); this is the simulation of the plant. Similarly, the mix-variance can be approximated by

$$
\|x(\cdot, t)\|_{H^{-\frac{1}{2}}}^{2} \approx \frac{1}{2} X^{\prime}(t) Q X(t),
$$

where $Q$ is symmetric and positive definite, denoted $Q=Q^{\prime} \succ 0$, and is defined in Appendix A.

The choice of $n$ is clearly important because if it's too high, computational power is being wasted, whereas if $n$ is too low, energy in wavenumbers larger than $n / 2$ will appear erroneously in wavenumbers less than $n / 2$ in a phenomenon known as aliasing [26]. This destabilizes the simulation. The larger the value of the diffusivity $\kappa$, the smaller $n$ needs to be because the diffusion removes the signal energy before it can cascade to the high wavenumbers [16]. Currently no theoretical bounds relating the accuracy of the solution to $n$ and $\kappa$ exist. To check whether $n$ is large enough to get acceptable simulation results for a given input sequence, it is necessary to check that increasing $n$ keeps the solution the same to some tolerance [28].

Assuming that $n$ is large enough for $\left(A_{\kappa}, N_{0}, N\right)$ to accurately approximate (2), part 3 of Proposition 1 suggests that that if $X(0)=\bar{x}_{0} \mathbf{1}_{n^{2}}$ then $X(t)=\bar{x}_{0} \mathbf{1}_{n^{2}}$ for all $t \in[0, \infty)$ where $\mathbf{1}_{n} \in \mathbb{R}^{n}$ is a vector of ones. This implies that if $X(0)=\bar{x}_{0} \mathbf{1}_{n^{2}}$, then

$$
\begin{aligned}
\frac{\mathrm{d} X}{\mathrm{~d} t}(t) & \approx 0 \\
\Longrightarrow\left(A_{\kappa}+N_{0}\right) \mathbf{1}_{n^{2}}+u(t) N \mathbf{1}_{n^{2}} & \approx 0 \text { for all }-1 \leq u(t) \leq 1, t \in[0, \infty) .
\end{aligned}
$$

For this condition to hold for all feasible $u(t)$, it must hold for $u(t)=0$, hence $\left(A_{\kappa}+N_{0}\right) \mathbf{1}_{n^{2}} \approx 0$. The case where $u(t)=1$ then implies $N \mathbf{1}_{n^{2}} \approx 0$. Hence it is reasonable to expect that $A_{\kappa}+N_{0}$ and $N$ will have eigenvalues at, or very close to, the origin with corresponding eigenvectors $\mathbf{1}_{n^{2}}$. This implies that the origin could be a marginally stable equilibrium point thus prohibiting the use of certain control system design methodologies, including the one considered below. To address this issue, we simplify the model used for the simulation of the plant for control purposes by exploiting properties discussed in Section 2.1 and the fact that we are only considering initial conditions with $\bar{x}_{0}=0$. From Proposition 1 we know that the approximation of the dynamics should satisfy

$$
\sum_{i=1}^{n^{2}} X_{i}(t) \approx \sum_{i=1}^{n^{2}} X_{i}(0), \quad \forall t \in[0, \infty)
$$

representing the fact that the dynamics conserve the mean of the scalar field. In the optimal control problem, we have assumed zero mean initial conditions. Therefore, it is reasonable to eliminate the last state using the relation

$$
X_{n^{2}}(t)=-\sum_{i=1}^{n^{2}-1} X_{i}(t), \quad \forall t \in[0, \infty) .
$$

The resultant reduced state vector, denoted $\bar{X}(t) \in \mathbb{R}^{n^{2}-1}, \bar{X}(t):=\left[I_{n^{2}-1} 0_{\left(n^{2}-1\right) \times 1}\right] X(t)$, where $I_{q} \in \mathbb{R}^{q \times q}$ is the identity matrix and $0_{n \times m} \in \mathbb{R}^{n \times m}$ is a matrix of zeros, then evolves with $t$ 
according to the realization $\left(\bar{A}_{\kappa}, \bar{N}_{0}, \bar{N}\right), \bar{A}_{\kappa}, \bar{N}_{0}, \bar{N} \in \mathbb{R}^{\left(n^{2}-1\right) \times\left(n^{2}-1\right)}$, where

$$
\begin{aligned}
\bar{A}_{\kappa} & :=\left[I_{n^{2}-1} 0_{\left(n^{2}-1\right) \times 1}\right] A\left[\begin{array}{c}
I_{n^{2}-1} \\
-\mathbf{1}_{n^{2}-1}^{\prime}
\end{array}\right], \\
\bar{N}_{0} & :=\left[I_{n^{2}-1} 0_{\left(n^{2}-1\right) \times 1}\right] N_{0}\left[\begin{array}{c}
I_{n^{2}-1} \\
-\mathbf{1}_{n^{2}-1}^{\prime}
\end{array}\right], \\
\bar{N} & :=\left[I_{n^{2}-1} 0_{\left(n^{2}-1\right) \times 1}\right] N\left[\begin{array}{c}
I_{n^{2}-1} \\
-\mathbf{1}_{n^{2}-1}^{\prime}
\end{array}\right] .
\end{aligned}
$$

Likewise, the cost matrix $\bar{Q}$ is defined as

$$
\bar{Q}:=\left[I_{n^{2}-1}-\mathbf{1}_{\left(n^{2}-1\right) \times 1}\right] Q\left[\begin{array}{c}
I_{n^{2}-1} \\
-\mathbf{1}_{n^{2}-1}^{\prime}
\end{array}\right] .
$$

For this reduced model $\left(\bar{A}_{\kappa}, \bar{N}_{0}, \bar{N}\right)$, the origin represents the approximation to the zero-mean subspace. Therefore, from Proposition 1 we know that the state field should converge to the origin for all feasible input sequences. If $n$ is large enough for the model dynamics $\left(\bar{A}_{\kappa}, \bar{N}_{0}, \bar{N}\right)$ to approximate the real plant, then the origin should be a globally asymptotically stable equilibrium point for all feasible input sequences. Considering the case where $u=0$ for all $t$, this implies that $\bar{A}_{\kappa}+\bar{N}_{0}$ must be Hurwitz. For a given $n$, this can be easily confirmed numerically. The matrix $\bar{A}_{\kappa}+\bar{N}_{0}$ being Hurwitz implies the origin is a globally asymptotically stable equilibrium point for the open-loop system, facilitating the use of the control design methodology considered below.

\subsection{Feedback control of bilinear systems}

The mixing control problem, denoted $\mathcal{P}\left(\bar{Q}, r, \bar{A}_{\kappa}, \bar{N}_{0}, \bar{N}\right)$, considered in this work is a single constrained input, homogeneous-in-the-state, bilinear quadratic regulator (BQR) problem written thus:

$$
\min _{\bar{X}(\cdot), u(\cdot)} \int_{0}^{\infty} \frac{1}{2} \bar{X}(t)^{\prime} \bar{Q} \bar{X}(t)+\frac{1}{2} r u(t)^{2} \mathrm{~d} t
$$

subject to $\bar{X}:[0, \infty) \rightarrow \mathbb{R}^{n^{2}-1}$ being the state trajectory of the realization $\left(\bar{A}_{\kappa}, \bar{N}_{0}, \bar{N}\right)$ with initial condition $\bar{X}_{i}(0)=x\left(v_{i}, 0\right)$ for $i=1, \ldots, n^{2}-1$ and

$$
-1 \leq u(t) \leq 1, \forall t \in[0, \infty)
$$

where $\bar{Q} \succ 0$ and $r>0$. This is the spatially discretized approximation of (5). To date this problem has no explicit solution, but an approach to find well-performing solutions does exist. We explore the properties of this approximation.

Proposition 2. [18, Thm. 6.2] Consider the problem $\mathcal{P}_{\text {app }}(\bar{Q}, r, \bar{A}, \bar{N}, \bar{B})$ defined by

$$
\min _{\bar{X}(\cdot), u(\cdot)} \int_{0}^{\infty} \frac{1}{2} \bar{X}(t)^{\prime} \bar{Q} \bar{X}(t)+\frac{1}{2} r u(t)^{2}+g(\bar{X}(t)) \mathrm{d} t,
$$


subject to

$$
\begin{array}{rr}
\frac{\mathrm{d}}{\mathrm{d} t} \bar{X}(t)=\bar{A} \bar{X}(t)+u(t) \bar{N} \bar{X}(t)+\bar{B} u(t), & \bar{X}(0)=\bar{X}_{0}, \\
u(t) \in[-1,1], & \forall t \in[0, \infty),
\end{array}
$$

where $\bar{Q} \succ 0, r>0, \bar{Q}, \bar{A}, \bar{N} \in \mathbb{R}^{\left(n^{2}-1\right) \times\left(n^{2}-1\right)}, \bar{B} \in \mathbb{R}^{n^{2}-1}, \bar{A}$ is Hurwitz and $g: \mathbb{R}^{n^{2}-1} \rightarrow[0, \infty)$, defined

$$
\begin{aligned}
& g(\bar{X}):=\left\{\begin{array}{cc}
|\zeta(\bar{X})|-\frac{1}{2} r, & |\zeta(\bar{X})|>r, \\
\frac{1}{2 r} \zeta(\bar{X})^{2}, & |\zeta(\bar{X})| \leq r,
\end{array}\right. \\
& \zeta(\bar{X}):=\bar{X}^{\prime} \bar{N}^{\prime} Z \bar{X}+\bar{B}^{\prime} Z \bar{X},
\end{aligned}
$$

with $Z \in \mathbb{R}^{\left(n^{2}-1\right) \times\left(n^{2}-1\right)}, Z \succ 0$, satisfying the Lyapunov equation

$$
\bar{A}^{\prime} Z+Z \bar{A}+\bar{Q}=0 .
$$

The solution to this problem, denoted $u^{*}(\cdot)$, can be written as a feedback law

$$
u^{*}(t)=\mu^{*}(\bar{X}(t)):=-\operatorname{sat}\left(\frac{1}{r} \zeta(\bar{X}(t))\right)
$$

where sat : $\mathbb{R} \rightarrow[-1,1]$ is the saturation function

$$
\operatorname{sat}(p):=\left\{\begin{array}{cl}
p & \text { if }|p| \leq 1 \\
p /|p| & \text { otherwise }
\end{array}\right.
$$

We shall refer to the solution of $\mathcal{P}_{\text {app }}(\bar{Q}, r, \bar{A}, \bar{N}, \bar{B})$ as the approximate bilinear regulator (aBQR) solution. Notice that this feedback solution is time-invariant and that it is unique [18]. The solution of $\mathcal{P}_{\text {app }}(\bar{Q}, r, \bar{A}, \bar{N}, 0)$ is shown to be a good approximation to that of $\mathcal{P}(\bar{Q}, r, \bar{A}, 0, \bar{N})$ for a number of numerical examples in [18], although to date no bounds on performance exist. In this fashion, the fluid mixing problem $\mathcal{P}\left(\bar{Q}, r, \bar{A}_{\kappa}, \bar{N}_{0}, \bar{N}\right)$ can be approximated by $\mathcal{P}_{\text {app }}\left(\bar{Q}, r, \bar{A}_{\kappa}+\bar{N}_{0}, \bar{N}, 0\right)$ and so a control law can be easily computed. It cannot be approximated by $\mathcal{P}_{\text {app }}\left(Q, r, A_{\kappa}+N_{0}, N, 0\right)$ because $A_{\kappa}+N_{0}$ is not Hurwitz, as discussed in Section 3.1. Note that as a result of projecting onto the zero mean subspace, the controller will have one less input than the state dimension of the simulation of the plant, i.e. $n^{2}-1$. The last state in the state vector is redundant and so the inputs to the controller are $\bar{X}(t):=\left[I_{n^{2}-1} 0_{\left(n^{2}-1\right) \times 1}\right] X(t)$. The closed-loop now takes the form shown in Figure 3.

Note also that $\bar{B}=0$ in the mixing of fluids problem. This reflects the fact that once at the origin, i.e. once the fluid has arrived at its homogeneous distribution, no amount of stirring can unmix it.

\subsection{Physical interpretation of the aBQR solution}

The aBQR solution is the optimal control law for a BQR problem with the stage cost appended by a non-negative term. We now consider the physical interpretation of this modified cost. Consider the function $V: \mathbb{R}^{n^{2}-1} \rightarrow[0, \infty)$ defined as

$$
V(\bar{X}(t)):=\bar{X}^{\prime}(t) Z \bar{X}(t),
$$




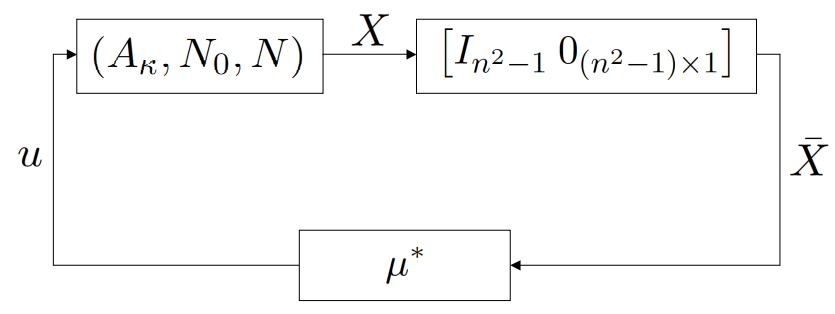

Figure 3: Feedback arrangement where $\mu^{*}: \mathbb{R}^{n^{2}-1} \rightarrow[-1,1]$ solves $\mathcal{P}_{\text {app }}\left(\bar{Q}, r, \bar{A}_{\kappa}+\bar{N}_{0}, \bar{N}, 0\right)$

where $Z \succ 0, Z \in \mathbb{R}^{\left(n^{2}-1\right) \times\left(n^{2}-1\right)}$ is the solution to (10) with $\bar{A}:=\bar{A}_{\kappa}+\bar{N}_{0}$. It is easy to show that

$$
u(\tau)=0, \forall \tau \in[t, \infty) \Longrightarrow \int_{t}^{\infty} \bar{X}^{\prime}(\tau) \bar{Q} \bar{X}(\tau) \mathrm{d} \tau=\bar{X}^{\prime}(t) Z \bar{X}(t),
$$

see for example [29, p. 85]. In other words, $V(\bar{X}(t))$ is the cost endured by going from $\bar{X}(t)$ to the origin with $u=0$, or the open-loop cost-to-go. The Lie derivative of $V$ tells us the instantaneous rate of decrease in this function:

$$
\dot{V}(\bar{X}(t))=2 \bar{X}(t)^{\prime}\left(\bar{A}_{\kappa}+\bar{N}_{0}+u(t) \bar{N}\right)^{\prime} Z \bar{X}(t) .
$$

Therefore the $\bar{X}^{\prime}(t) \bar{N}^{\prime} Z \bar{X}(t)$ term can be seen as a measure of how effectively the input can decrease the open-loop cost-to-go. By appending the stage cost with a non-negative function of $\left|\bar{X}^{\prime}(t) \bar{N}^{\prime} Z \bar{X}(t)\right|$ we are penalizing modes where this quantity is large. That is, we are weighting against steering the system to places where the control will be particularly influential. In reality the optimal solution may want to encounter these states.

Some interesting conclusions can also be drawn by considering the nature of the proposed feedback solution. It makes sense to consider when $\bar{X}^{\prime}(t) \bar{N}^{\prime} Z \bar{X}(t)$ is going to be large. If $\bar{N} \bar{X}(t)=$ 0 , e.g. when a blob of dye lies in the center of the stirring motion, then the control input will be zero. This makes sense because, at that instant, the velocity field will have no effect. Likewise, if the concentration field is already well mixed, $Z \bar{X}(t)$ will be small, hence the input will be small. If $\bar{N} \bar{X}(t)$ and $Z \bar{X}(t)$ are parallel, $\bar{X}^{\prime}(t) \bar{N}^{\prime} Z \bar{X}(t)$ will be large and so $u(t)$ will have saturated. In physical terms, this means if the effect of the velocity field is to move $\bar{X}(t)$ away from a field for which the open-loop cost-to-go is large, then the input will be large. All these properties make physical sense.

\subsection{Dependence on model diffusivity}

There have been remarks in the literature suggesting the selection of a well-performing input sequence is relatively insensitive to differences between the model and plant diffusivities [16, 30]. As discussed in Section 3.1, the larger the model diffusivity, the simpler the model. This suggests that a simple model based on a high diffusivity model of the plant can be used for control system design. To begin to understand the role of diffusivity in the aBQR controller design, consider the case where $f_{1}=0$, therefore $\bar{N}_{0}=0$. Scaling the diffusivity $\kappa$ by $\alpha$ results in $\bar{A}_{\kappa} \rightarrow \bar{A}_{\alpha \kappa}=\alpha \bar{A}_{\kappa}$. For details of why this is the case, see the definition of $A_{\kappa}$ in Appendix A. Consider the following result: 
Proposition 3. The feedback law solution to $\mathcal{P}_{\text {app }}\left(\bar{Q}, r, \bar{A}_{\kappa}, \bar{N}, 0\right)$ is also the solution to $\mathcal{P}_{\text {app }}\left(\alpha \bar{Q}, r, \alpha \bar{A}_{\kappa}, \bar{N}, 0\right)$ for any $\alpha>0$.

Proof. The aBQR solution to $\mathcal{P}_{\text {app }}\left(\alpha \bar{Q}, r, \alpha \bar{A}_{\kappa}, \bar{N}, 0\right)$ is

$$
u^{*}(t)=\mu^{*}(\bar{X}(t))=-\operatorname{sat}\left(\frac{1}{r} \bar{X}(t)^{\prime} \bar{N}^{\prime} \hat{Z} \bar{X}(t)\right)
$$

where $\hat{Z}$ satisfies

$$
\alpha \bar{A}_{\kappa}^{\prime} \hat{Z}+\alpha \hat{Z} \bar{A}_{\kappa}+\alpha \bar{Q}=0
$$

Clearly the solution $\hat{Z}$ is independent of $\alpha$, thus confirming the result.

The interesting point to note here is that for the control law designed to be invariant to diffusivity, the cost matrix $\bar{Q}$ must also be scaled by $\alpha$. This has not been noted previously, purely because in both [16] and [30] there is no input penalization, hence the optimal input is bangbang [18] and so depends only on the switching instances and not the magnitude of the switching function inbetween zero-crossings. When $f_{1}=0$, there is no base field; obviously this case is trivial. What Proposition 3 does not tell us is what the effect of scaling diffusivity is in the case where there is a base flow, i.e. $f_{1} \neq 0$.

\subsection{Controller design}

The aim of the work is to design a controller such that, when placed in closed-loop with the simulation of the plant, the system behaves well with respect to the BQR cost (8a). The work of [18] suggests that the feedback solution to $\mathcal{P}_{\text {app }}\left(\bar{Q}, r, \bar{A}_{\kappa}+\bar{N}_{0}, \bar{N}, 0\right)$ should be a good approximation to that of $\mathcal{P}\left(\bar{Q}, r, \bar{A}_{\kappa}, \bar{N}_{0}, \bar{N}\right)$. Likewise, observations from [16] and [30] suggest that the control law solving $\mathcal{P}\left(\bar{Q}, 0, \alpha \bar{A}_{\kappa}, \bar{N}_{0}, \bar{N}\right)$ could be a good approximation to that of $\mathcal{P}\left(\bar{Q}, 0, \bar{A}_{\kappa}, \bar{N}_{0}, \bar{N}\right)$. Combining these observations with the concepts from Section 3.4, we propose that the solution $\mu_{\alpha, \beta}^{*}, \alpha \geq 1, \beta>0$ to $\mathcal{P}_{\text {app }}\left(\beta Q, r, \alpha \bar{A}_{\kappa}+\bar{N}_{0}, \bar{N}, 0\right)$ may approximate that of $\mathcal{P}\left(Q, r, A_{\kappa}, N_{0}, N\right) . \alpha$ represents the ratio of model to plant diffusivity. As discussed in Section 3.4, the larger the model diffusivity the simpler the model. We are interested in designing a control law for simpler models than that of the plant and so only consider $\alpha \geq 1$. There are two approximations associated with such a design:

1. Model-plant mismatch resulting from different diffusivities;

2. Stage cost mismatch between the aBQR and BQR problem formulations.

Increasing $\alpha$ increases the model-plant mismatch. It will also affect the ratio between the three terms in the stage cost, i.e. the state penalizing term, the input penalizing term and the 'regularizing' term $g$, because the open-loop cost-to-go will depend upon the model diffusivity. Tuning $\beta$ can then attempt to redress this balance. In the numerical example we investigate the effects of $\alpha$ and $\beta$ on the performance with respect to the BQR cost. The aim is to find an $(\alpha, \beta)$ pair to achieve acceptable performance. Note that interestingly and initially counter-intuitively, it could be that the introduction of model-plant mismatch and amendment of the cost may improve performance of the induced controller. Clearly the model-plant mismatch will not improve performance with respect to the aBQR cost, but might with respect to the BQR cost. 


\section{Numerical example}

In this section we use a numerical example to investigate the effects of the relevant design decisions discussed in Section 3.5. We implement the scheme on a numerical example from the literature $[15,31]$ representative of an experiment where a set of magnets are used to generate an array of counter-rotating vortices by magnetohydrodynamic effects. The kinematics corresponding to this setup can be captured by the velocity fields

$$
\begin{aligned}
& f_{1}(y):=\left[-\sin \left(2 \pi y_{1}\right) \cos \left(2 \pi y_{2}\right) \cos \left(2 \pi y_{1}\right) \sin \left(2 \pi y_{2}\right)\right]^{\prime}, \\
& f_{2}(y):=\left[-\cos \left(2 \pi y_{1}\right) \sin \left(2 \pi y_{2}\right) \sin \left(2 \pi y_{1}\right) \cos \left(2 \pi y_{2}\right)\right]^{\prime} .
\end{aligned}
$$

The initial field, representing a large drop of concentration as shown in Figure 11(a), is defined as

$$
x_{0}(y):=\left\{\begin{array}{cc}
\sin \left(2 \pi y_{1}\right) \sin \left(2 \pi y_{2}\right)-\frac{1}{4 \pi^{2}} & \forall y \in[0,0.5]^{2}, \\
-\frac{1}{4 \pi^{2}} & \forall y \in \mathcal{D} \backslash[0,0.5]^{2} .
\end{array}\right.
$$

The constant offset $-\frac{1}{4 \pi^{2}}$ ensures that $\bar{x}_{0}=0$. This example may seem a trivial case because the velocity fields contain just a single scale. However, in his celebrated paper [5], Aref reports that very regular and simple flows can lead to highly irregular advection patterns.

It is possible to design a controller for a model based upon a coarser discretization than that of the simulation of the plant. Let the controller be designed for a model with a discretization to $m^{2}$ states, $m<n$. The controller therefore has $m^{2}-1$ inputs that are an approximation of the simulation state at the $m^{2}-1$ points equispaced across the domain in the $m^{2}$-tuple $\mathcal{Y}_{m}$ (defined in Section 3.1) without the $\left(\mathrm{m}^{2}\right)^{\text {th }}$ element (because of the projection to the zero mean subspace). However, it is not appropriate to simply interpolate the state field at the points in $\mathcal{Y}_{m}$ to get these values, because $m$ may be too small to capture the effects of all the state field frequency components. As a result, there may be aliasing. Instead, we filter the state field from the simulation of the plant to contain only the first $m / 2$ Fourier modes in each direction and then sample at the $m^{2}$ locations in $\mathcal{Y}_{m}$. Let $S_{m} \in \mathbb{R}^{m^{2} \times n^{2}}$ denote this linear sampling and filtering operation; see Appendix B for the definition. The feedback law is then written

$$
u(t)=\mu_{\alpha, \beta}^{*}\left(\left[I_{m^{2}-1} 0_{\left(m^{2}-1\right) \times 1}\right] S_{m} X(t)\right),
$$

where $\mu_{\alpha, \beta}^{*}: \mathbb{R}^{m^{2}-1} \rightarrow[-1,1]$ is the controller solving $\mathcal{P}_{\text {app }}\left(\beta \tilde{Q}, r, \alpha \tilde{A}_{0}+\tilde{N}_{0}, \tilde{N}, 0\right)$, where $\tilde{A}_{0}, \tilde{N}_{0}, \tilde{N}, \tilde{Q} \in$ $\mathbb{R}^{\left(m^{2}-1\right) \times\left(m^{2}-1\right)}$ are the system and cost matrices for a discretized model with $m^{2}$ states projected onto the zero mean subspace. The effect of suboptimality introduced by this change of discretization is examined in the numerical example.

The proposed control law is defined such that the input depends on the current simulation state. In practice this is not feasible because it takes some time to measure the state and compute the control input and this can only be done at a discrete set of sample instants. To account for this, the control law is implemented with a zero-order sample-and-hold, i.e.

$$
u(t)=\mu_{\alpha, \beta}^{*}\left(\left[I_{m^{2}-1} 0_{\left(m^{2}-1\right) \times 1}\right] S_{m} X\left(\left\lfloor\frac{t}{T_{s}}\right\rfloor T_{s}\right)\right),
$$

where $T_{s}$ is the sample period and $\lfloor\cdot\rfloor$ denotes the floor function.

In Section 4.1 we compare the aBQR solution with $\alpha=\beta=1$ to a locally optimal, open-loop solution, to get an idea of the degradation in performance introduced by the aBQR solution. In 
Section 4.2 we investigate the effects of coarseness of discretization of the model about which the aBQR solution is designed on the closed-loop performance. In Section 4.3 we discuss the relevant trade-offs associated with designing the aBQR controller for a model with a different diffusivity to that of the plant. Section 4.4 details a comparison between the performance of the closed-loop controller to its open-loop counterpart in the presence of disturbances. We conclude the section by analyzing the physical properties of the closed-loop system in Section 4.5.

The simulation of the plant has $52 \times 52$ states for all of the numerical test cases, i.e. $n=52$ with $n$ chosen to satisfy the conditions in Section 3.1, and the numerical integration is performed by SUNDIALS [32]. The control laws are computed with $r=10^{-4}$. The zero-order-hold has a sample time $T_{s}=0.002 \mathrm{~s}$.

\section{1. aBQR performance versus a locally optimal solution}

With plant diffusivity $\kappa=0.15 \mathrm{~s}^{-1}$, a model of the plant exists that is small enough for a locally optimal solution to the $\mathrm{BQR}$ problem to be approximated by finding a numerical solution to

$$
\left(\tilde{X}^{*}(X(0), \cdot), u^{*}(X(0), \cdot)\right):=\arg \min _{\tilde{X}(\cdot), u(\cdot)} \int_{0}^{T} \frac{1}{2} \tilde{X}(t)^{\prime} \tilde{Q} \tilde{X}(t)+\frac{1}{2} r u(t)^{2} \mathrm{~d} t
$$

subject to

$$
\begin{aligned}
\frac{\mathrm{d} \tilde{X}}{\mathrm{~d} t}(t) & =\left(\tilde{A}_{\kappa}+\tilde{N}_{0}\right) \tilde{X}(t)+u(t) \tilde{N} \tilde{X}(t), \\
\tilde{X}(0) & =\left[\begin{array}{ll}
I_{m^{2}-1} & 0_{\left(m^{2}-1\right) \times 1}
\end{array}\right] S_{m} X(0), \\
u(t) & \in[-1,1], \quad \forall t \in[0, T] .
\end{aligned}
$$

Details of the approximation procedure can be found in Appendix C. The solution to the nonlinear program is computed with IPOPT [33] called from MATLAB 7.4. A horizon of $T=10 \mathrm{~s}$ is sufficiently long for the initial field to be almost completely mixed. The open-loop input applied to the simulation of the plant is $u(t)=u^{*}\left(X(0),\left\lfloor\frac{t}{T_{s}}\right\rfloor T_{s}\right)$ for all $t \in[0,10]$. We judge suboptimality of the aBQR solution designed with $\alpha=\beta=1$ by comparing the closed-loop aBQR solution to the open-loop locally optimal solution. Figure 4 shows the results for the $m=8,10,12$ cases. Although the input sequences from the two control approaches are similar, they are not identical. The degradation of performance is only very small. This supports conclusions from [16] that there are many well performing input sequences all achieving similar costs and from Section 3 that the aBQR solution can be a good approximation to $\mathcal{P}\left(Q, r, A_{\kappa}, N_{0}, N\right)$. Computational constraints restrict the locally optimal control law to being computed with $N_{c}=100$ corresponding to first-order holds in the computation of length $T_{u}=5 / 99 \mathrm{~s}$.

\subsection{Effect of the coarseness of model discretization}

In this section we investigate the effect of model fidelity on closed-loop performance. We consider the case where $\kappa=0.05 \mathrm{~s}^{-1}$ because approximately $40 \times 40$ states (i.e. $m=40$ ) are needed to accurately represent the input-output behavior and this is close to the limit of the size of Lyapunov equations that can be easily solved in MATLAB with the lyap function. Figure 5 shows the inputs and costs for varying degrees of coarseness of discretization of the model, with $\alpha=\beta=1$, about which the controller is designed. Interestingly, the closed-loop input and cost are reasonably insensitive to this coarseness. For the $m=10,16$ cases, this is significantly lower than the fidelity required for the simulation model with $n=52$. 


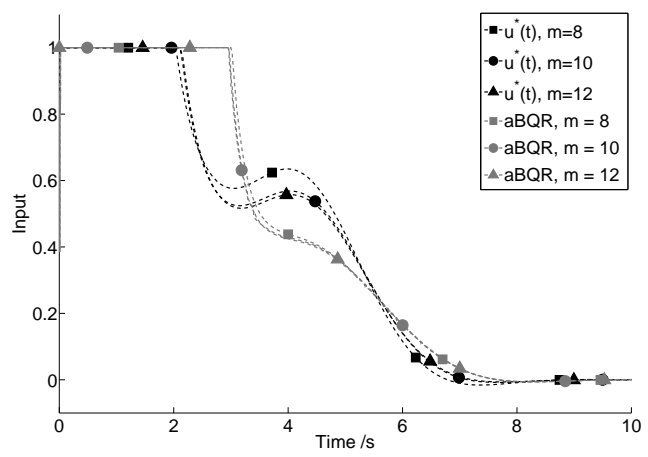

(a) Input

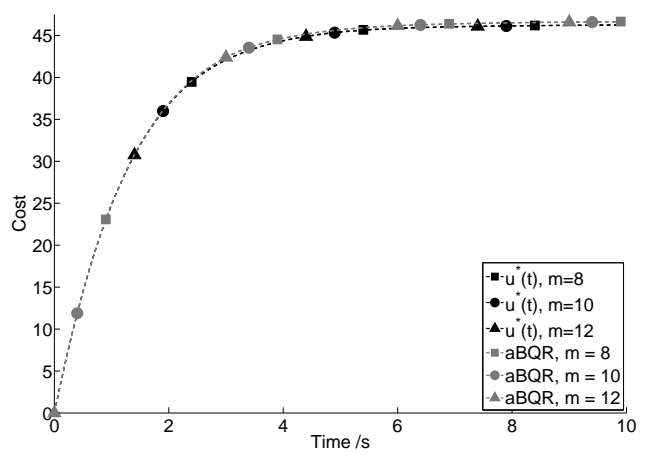

(b) BQR Cost

Figure 4: Comparison of optimal and aBQR input sequences for a simulation of the plant with diffusivity $\kappa=0.15 \mathrm{~s}^{-1}$

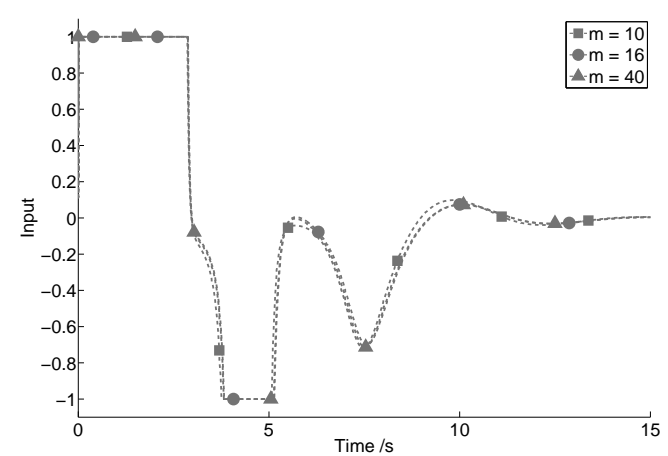

(a) Input

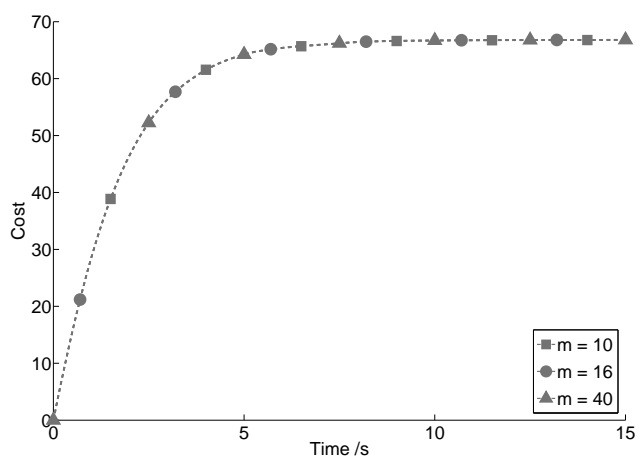

(b) BQR Cost

Figure 5: Comparison of aBQR performance for a range of discretizations. $\kappa=0.05 \mathrm{~s}^{-1}$.

\subsection{Effect of $\alpha$ and $\beta$ on the aBQR solution}

The two parameters defining the aBQR controller are the model diffusivity scaling factor $\alpha$ and the parameter by which one has to scale the $\operatorname{cost} \beta$. We consider the tuning of these parameters for three different plant diffusivities; $\kappa=0.15 \mathrm{~s}^{-1}, 0.05 \mathrm{~s}^{-1}, 0.005 \mathrm{~s}^{-1}$. These will be referred to as the high, medium and low diffusivity cases, respectively. To ensure accurate computation of the cost, we simulate the three cases for $15 \mathrm{~s}, 20 \mathrm{~s}$ and $25 \mathrm{~s}$, respectively. We set $m$ to be significantly larger than is required for the closed-loop performance to be independent of further increases in fidelity so any discretization effects will be negligible. Hence we use $m=16,22,32$ for the high, medium and low diffusivity cases, respectively. The model for simulation has $n=52$ throughout. In Section 3.4 it was shown that when $\bar{N}_{0}=0$, i.e. there is no base flow, $\mu_{\alpha, \alpha}^{*}$ is independent of $\alpha$. First we investigate the effects of extending this relation to the case where $\bar{N}_{0} \neq 0$ by designing $\mu_{\alpha, \alpha}^{*}$ with a range of values of $\alpha$ for the given example. Figure 6 shows the closed-loop simulation results. In all cases, the closed-loop BQR performance varies by $<5 \%$ over the ranges considered, supporting conclusions that the performance is particularly insensitive to changes in model diffusivity. For the high and medium diffusivity cases, increasing $\alpha$ increases the BQR cost. However, for the low diffusivity case, increasing $\alpha$ greater than one initially results in a decrease. Although the maximum 


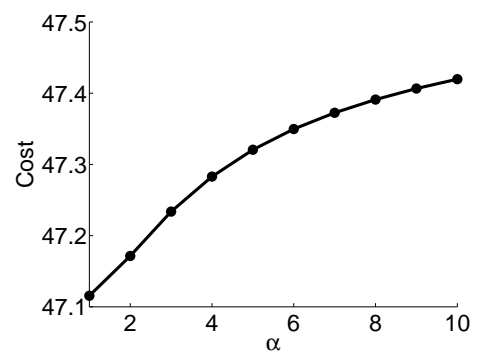

(a) Cost at $t=15 \mathrm{~s}, \kappa=0.15 \mathrm{~s}^{-1}$

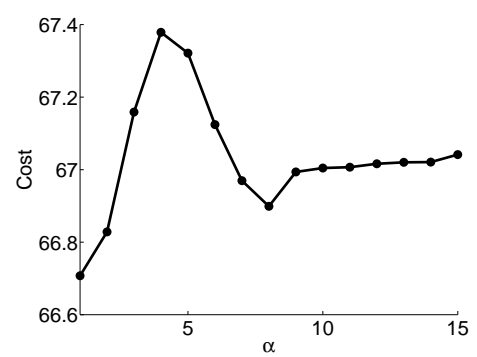

(b) Cost at $t=20 \mathrm{~s}, \kappa=0.05 \mathrm{~s}^{-1}$

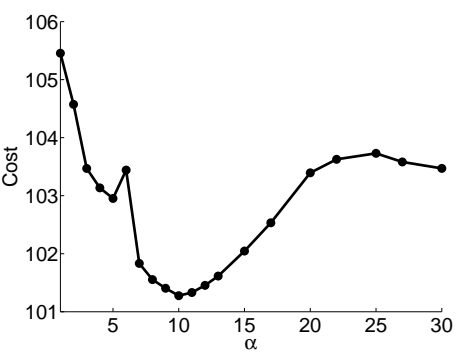

(c) Cost at $t=25 \mathrm{~s}, \kappa=0.005 \mathrm{~s}^{-1}$

Figure 6: BQR Cost versus $\alpha, \beta=\alpha$ for three plant diffusivities.

decrease is only approximately $4 \%$, it still shows that additional performance with respect to the BQR cost can be obtained by increasing the model-plant mismatch. We now attempt to justify this phenomenon.

As discussed in Section 3.3, the non-negative term $g$ at state $\tilde{X}(t)=\left[I_{m^{2}-1} 0_{\left(m^{2}-1\right) \times 1}\right] S_{m} X(t)$ is a function of $\tilde{X}^{\prime}(t) \tilde{N}^{\prime} \tilde{Z} \tilde{X}(t)$. The matrix $\tilde{Z} \succ 0, \tilde{Z} \in \mathbb{R}^{\left(m^{2}-1\right) \times\left(m^{2}-1\right)}$ is the solution of (10) with $\bar{A}:=\alpha \tilde{A}_{\kappa}+\tilde{N}_{0}, \bar{Q}=\tilde{Q}$ and is such that $\tilde{X}^{\prime}(t) \tilde{Z} \tilde{X}(t)$ is the open-loop cost-to-go of the model $\left(\alpha \tilde{A}_{\kappa}, \tilde{N}_{0}, \tilde{N}\right)$ with cost matrix $\tilde{Q}$. It makes sense to hypothesize that the smaller the model diffusivity is, the larger the open-loop cost-to-go will be. For example, much less stirring is required to mix dye with water than chocolate cream with plain cream. Therefore, it is reasonable to conjecture that the larger the diffusivity, the smaller $\tilde{Z} \tilde{X}(t) . \tilde{N} \tilde{X}(t)$ is clearly independent of diffusivity and hence we expect $\tilde{X}^{\prime}(t) \tilde{N}^{\prime} \tilde{Z} \tilde{X}(t)$ to decrease with increasing diffusivity. The diffusivity has no effect on the instantaneous mixing cost $\tilde{X}^{\prime}(t) \tilde{Q} \tilde{X}(t)$ and so we suggest that an increase in model diffusivity will result in a decrease in the effect of the additional non-negative term $g$ on the stage cost. Obviously increasing the diffusivity introduces a model-plant mismatch, hence it cannot be scaled arbitrarily and so there is a trade-off.

Next we investigate the effects of designing a controller $\mu_{\alpha, \beta}^{*}$ with $\beta \neq \alpha$ for a range of $\alpha$ values and plant diffusivities. The results are shown in Figure 7. Again, the closed-loop performance is seen to be reasonably insensitive to such changes. In the high and medium diffusivity cases, reducing $\beta$ in the range $0.5 \alpha<\beta<\alpha$ causes a decrease in the performance $\approx 5 \%$. The performance seems to be even less sensitive to changes to $\beta>\alpha$, the worst case change being approximately $1 \%$. Obviously $\beta \gg \alpha(\beta \ll \alpha)$ will be penalizing the state mixedness much more (less) harshly than the input and so will perform poorly. The low diffusivity case is even less sensitive to changes in $\beta$ with variations of $<2 \%$ over a broader range. This simulation justifies the use of $\beta=\alpha$ for control design. Additional performance can be achieved with some tuning, but the benefits are only very small.

Having investigated the effect of model diffusivity on closed-loop performance, we now consider briefly its effect on the input. It is interesting to see whether the input sequences are similar or wildly different. We consider as a test case the medium diffusivity plant and $\beta=\alpha$ for a range of $\alpha$ values. The results are in Figure 8 . The inputs are very similar for small times, but interestingly Figure 8 shows there is a significant change in closed-loop input with $\alpha$, particularly for times greater than $5.5 \mathrm{~s}$. There seem to be two classes of solution: one with positive input, the other with negative input. This could be a result of the fact that even the global BQR solution is not unique for certain initial conditions; imagine dropping red dye into a tray of white and stirring in circles 


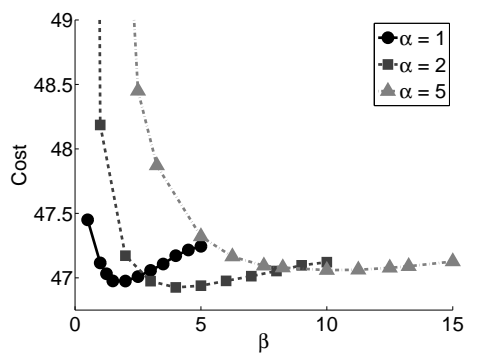

(a) Cost at $t=15 \mathrm{~s}, \kappa=0.15 \mathrm{~s}^{-1}$

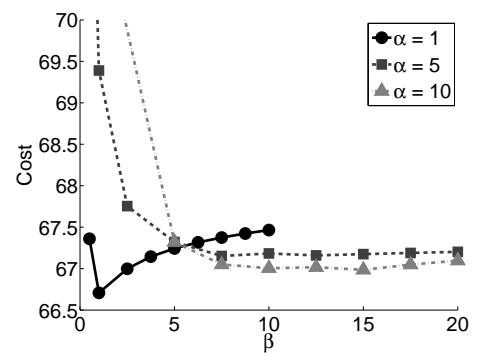

(b) Cost at $t=20 \mathrm{~s}, \kappa=0.05 \mathrm{~s}^{-1}$

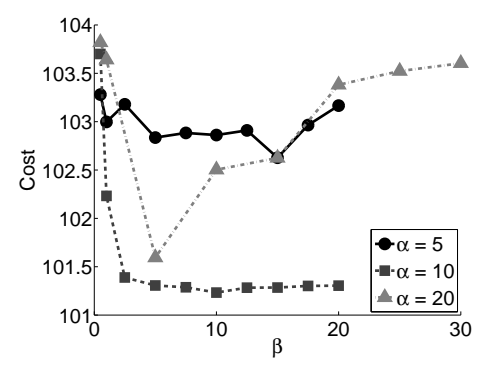

(c) Cost at $t=25 \mathrm{~s}, \kappa=0.005 \mathrm{~s}^{-1}$

Figure 7: BQR Cost versus $\beta$ for a range of $\alpha$ values for three plant diffusivities.

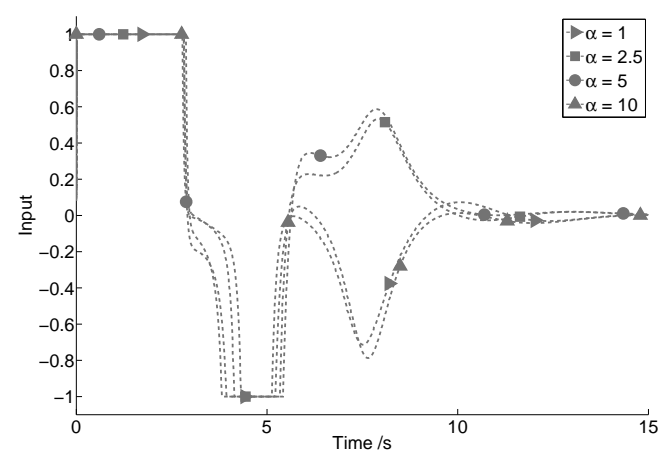

Figure 8: Input sequences for the aBQR closed-loop system for a range of values of $\alpha$ with $\beta=\alpha$.

— the mixing performance will be identical whether one stirs clockwise or counter-clockwise.

\subsection{Disturbance rejection}

A major benefit of a closed-loop approach is the ability to reject the effects of disturbances. These disturbances can take a number of forms:

1) Varying initial conditions. For example, if the aim was to use a fan to mix temperature on a microprocessor but one component had generated more heat than was expected;

2) Injection of inhomogeneity during an interval. It is reasonable to consider applications where not all the inhomogeneity is added in one go. For example, it may be that some of the chemicals are delayed in the pipe of a mixer;

3) Varying diffusivity. We have assumed constant diffusivity; however, with ambient temperature changes this may not be the case;

4) Velocity field mismatch. We have assumed Stokes' flow because it significantly simplifies the problem. However, in reality there will be some inertial forces and the switching of velocity fields may not be instantaneous. Likewise, there may be errors between the predicted velocity field induced by a certain forcing function and the real velocity field.

By designing a closed-loop strategy to reject the effects of these disturbances offers obvious advantages from a practical engineering viewpoint. We now briefly consider the effects of such disturbances on the numerical example. To do this, we ran the aBQR closed-loop for the interval 


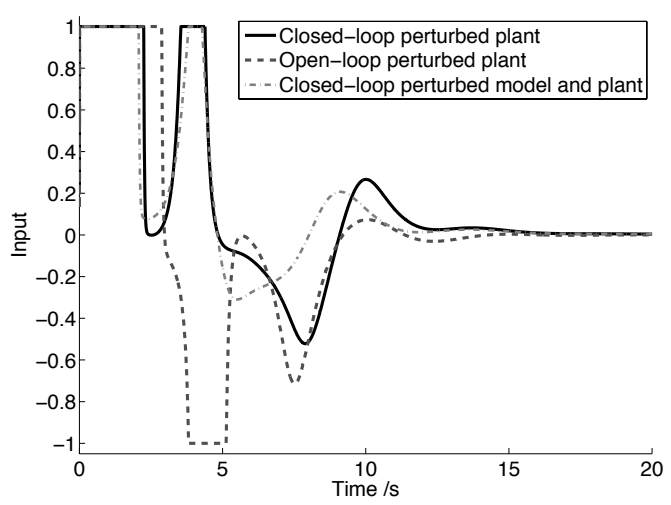

(a) Input

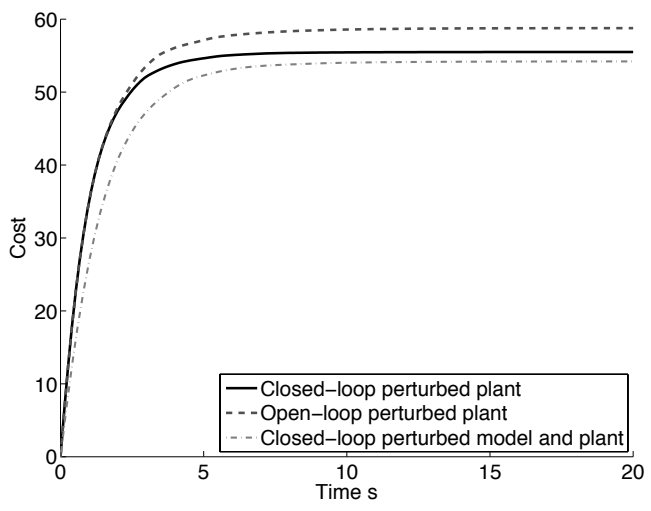

(b) BQR Cost

Figure 9: Simulations showing effect of model-plant mismatch on performance of open-loop and closed-loop solutions.

$[0,40] \mathrm{s}$ with initial condition $x_{0}$ and saved the closed-loop input sequence, denoted $u_{\mathrm{OL}}\left(t, x_{0}\right)$. Then, we perturbed the plant and compared the new closed-loop performance to that of the open-loop plant with the input $u(t)=u_{\mathrm{OL}}\left(t, x_{0}\right)$ applied.

Firstly, we look at the effects of a mismatch on the velocity fields and diffusivity. We consider the case where both the plant velocity fields are $10 \%$ stronger than anticipated and the base flow is perturbed:

$$
\begin{aligned}
f_{1}(y):= & 1.1\left[-\sin \left(2 \pi y_{1}\right) \cos \left(2 \pi y_{2}\right) \cos \left(2 \pi y_{1}\right) \sin \left(2 \pi y_{2}\right)\right]^{\prime} \\
& +0.2\left[-\cos \left(2 \pi y_{1}\right) \sin \left(2 \pi y_{2}\right) \sin \left(2 \pi y_{1}\right) \cos \left(2 \pi y_{2}\right)\right]^{\prime}, \\
f_{2}(y):= & 1.1\left[-\cos \left(2 \pi y_{1}\right) \sin \left(2 \pi y_{2}\right) \sin \left(2 \pi y_{1}\right) \cos \left(2 \pi y_{2}\right)\right]^{\prime},
\end{aligned}
$$

and the diffusivity is larger than expected, $\kappa=0.07 \mathrm{~s}^{-1}$. Figure 9 shows the costs and inputs for the closed-loop and open-loop cases when designed about the unperturbed plant. The closed-loop performance is seen to be approximately $9 \%$ better than the open-loop performance over a $20 \mathrm{~s}$ simulation. We also compare the performance to that of a controller designed for the perturbed plant. The improvement in performance is approximately $3 \%$ indicating that the system equipped with a controller designed around a nominal model can perform only marginally suboptimally in the face of a significant model-plant mismatch. Note that this is in stark contrast to the open-loop approach.

The second case we consider is where some of the inhomogeneity is added later in the interval, so $x(0)=x_{0}$, then at $t=5$ a further $0.5 x_{0}$ is added. Figure 10 shows the comparative performances over a 40s simulation. The closed-loop system reacts to the disturbance and so performs more than $30 \%$ better than its open-loop counterpart.

\subsection{Physical properties of aBQR solution}

To shed some light on the physical properties of the aBQR solution we show the velocity and scalar fields at a few time instants across the interval of interest for the case $\kappa=0.05 \mathrm{~s}^{-1}, \alpha=\beta=1$, $m=22$. Figure 11 shows the concentration field at a range of times with the instantaneous velocity fields overlaid. Notice from Figure 11(a) that the velocity field is such that the highest velocity gradient overlaps the concentration island. As time progresses, the concentration field begins 


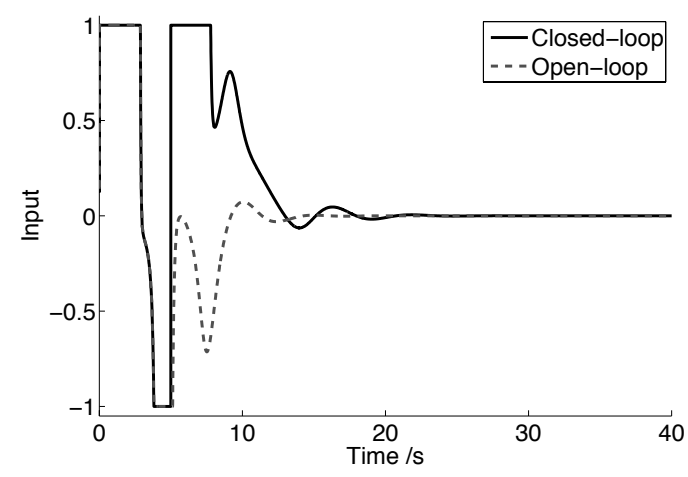

(a) Input

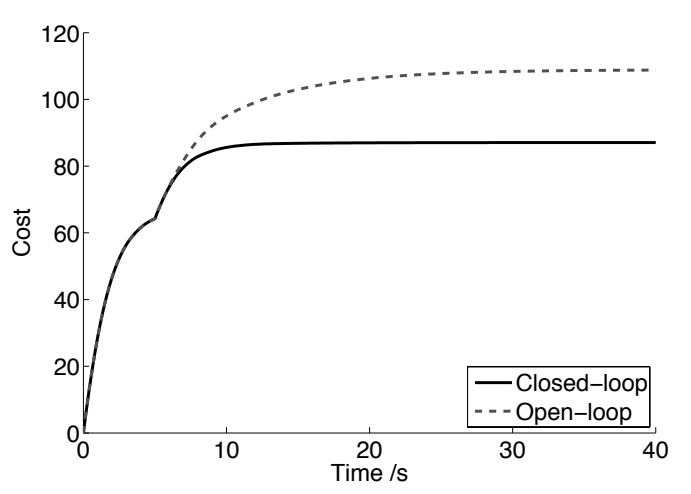

(b) BQR Cost

Figure 10: Simulations showing effect of late injection of inhomogeneity on performance of open-loop and closed-loop solutions.

to align with the velocity field with unmixed islands beginning to appear in regions with low velocity gradients. When these islands become approximately perpendicular to the streamlines of the $u=-1$ velocity field, the input switches, as in Figure 11(b). The saturated nature is reflective of the fact that in the early phases, the field is very poorly mixed. This first few seconds can be thought of as the flow stretching the blob while the next phase folds it. As the field becomes more mixed, and begins to align with this second flow the contribution of the input to the cost becomes notable and the input value moves from its extremes. Slight variations are then used to align islands with regions of velocity gradient and put concentration gradients perpendicular to the velocity field, as can be seen in Figure 11(c). This is not always the case because the aim is to minimize the integral of the stage cost, not the gradient of it at each time instant. The closed-loop behavior therefore shows many of the aspects one expects of a well mixing flow $[13,15,16]$.

\section{Conclusions}

The contribution of this work is to propose a novel, closed-loop solution relating velocity field to concentration field, for the promotion of mixing in a fluid flow. The control law is extremely simple, despite the complexity of the plant, and as such can be applied to real mixing applications. This and the fact that the closed-loop system will inherently reject disturbances are significant advantages of the proposed approach over existing open-loop methods. Although there are no theoretical performance guarantees, the method is shown to work well on a numerical example and the closed-loop velocity fields obey several features expected in a well mixing flow; closed-loop streamlines are shown to be such that regions of high spatial velocity gradient target unmixed islands and tend to be perpendicular to the contours of the scalar field. This goes some of the way to justifying the method's efficacy.

The controller is parameterized by $\alpha$ and $\beta$. These quantities represent the ratio of model to plant diffusivity and the amount by which the mixing term is scaled in the stage cost, respectively. In the numerical example, the closed-loop performance is shown to be reasonably insensitive to these parameters, provided $\beta$ is in the neighborhood of $\alpha$. This is interesting because it supports conclusions that a well-performing controller can be designed around a model with significantly larger diffusivity than that of the plant. The design process we advocate is: 


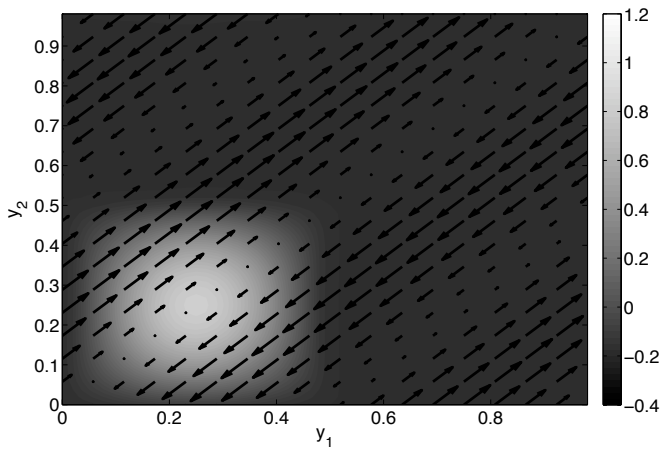

(a) $t=0.1 \mathrm{~s}$

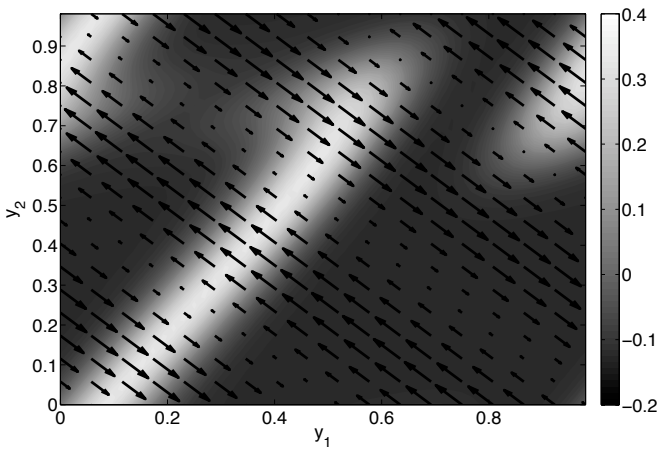

(b) $t=2.8 \mathrm{~s}$

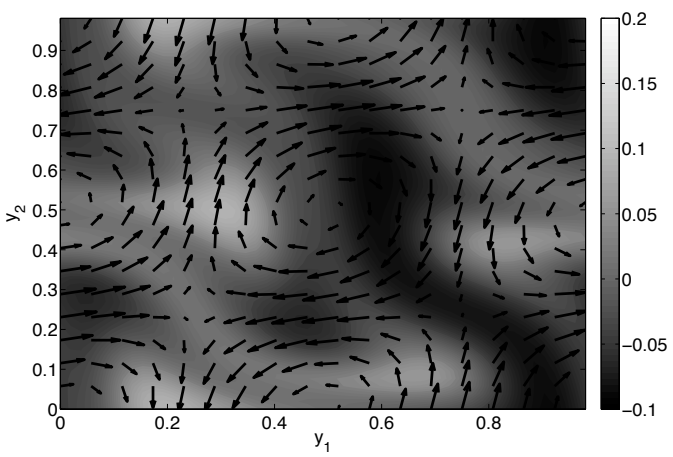

(c) $t=6 \mathrm{~s}$

Figure 11: Closed-loop evolution of a blob of dye. The instantaneous velocity fields are represented by the arrows, with the arrow length representing the speed of the flow at that point. The surface color represents the value of scalar field.

1. Pick the controller complexity $m$;

2. Pick $\alpha$ such that a controller can be designed around a model with diffusivity $\alpha \kappa$ and $m^{2}-1$ states;

3. Set $\beta=\alpha$;

4. Compute $\mu_{\alpha, \alpha}^{*}$;

5. Tune $\beta$ in the neighborhood $\beta \approx \alpha$ and compute $\mu_{\alpha, \beta}^{*}$ as required.

A direction for future work could be to attempt to extend this contribution to the multi-input case.

\section{Acknowledgments}

The authors would like to thank Professor John Christos Vassilicos for many useful discussions about the mixing of fluids and Juan Marcos Garcia de la Lopez for his help with generating Figure 1. 


\section{Appendix A. Discretization matrices}

The discretized realization is defined as

$$
\begin{aligned}
A_{\kappa} & :=\kappa\left(D_{1}^{2}+D_{2}^{2}\right), \\
N_{0} & :=-\operatorname{diag}\left(F^{11}\right) D_{1}^{1}-\operatorname{diag}\left(F^{12}\right) D_{2}^{1}, \\
N & :=-\operatorname{diag}\left(F^{21}\right) D_{1}^{1}-\operatorname{diag}\left(F^{22}\right) D_{2}^{1},
\end{aligned}
$$

where $F_{j n+k+1}^{a b}:=f_{b}^{a}\left(\left[\frac{j}{n} \frac{k}{n}\right]^{\prime}\right), a, b \in\{1,2\}$ and $D_{q}^{p} \in \mathbb{R}^{n^{2} \times n^{2}}, p \in \mathbb{Z}_{\geq 0}, q \in\{1,2\}$ are 2D differentiation matrices. For Fourier discretizations, these matrices are defined as

$$
\begin{aligned}
& D_{1}^{p}:=I_{n} \otimes D^{p}, \\
& D_{2}^{p}:=D^{p} \otimes I_{n},
\end{aligned}
$$

where $D^{p} \in \mathbb{R}^{n \times n}$ denotes the $p^{\text {th }}$-order Fourier differentiation matrix [27], $I_{n} \in \mathbb{R}^{n \times n}$ is the identity matrix and $\otimes$ is the Kronecker product.

$$
Q:=\hat{T}_{n}^{\prime} \operatorname{diag}(L) \hat{T}_{n},
$$

where $\hat{T}_{n} \in \mathbb{R}^{n \times n}$ represents the matrix-vector $2 \mathrm{D}$ discrete Fourier transform matrix defined $\hat{T}_{n}:=$ $\left(T_{n} \otimes I_{n}\right)\left(I_{n} \otimes T_{n}\right), T_{n}$ is the $n$-dimensional discrete Fourier transform matrix [27] and $L \in \mathbb{R}^{n^{2}}$ is defined as

$$
L_{(i-1) n+j+1}:=\left(1+(2 \pi)^{2}\left(\left(-\frac{n}{2}+j\right)^{2}+\left(-\frac{n}{2}+i\right)^{2}\right)\right)^{-\frac{1}{2}}, \quad i, j=1, \ldots, n .
$$

\section{Appendix B. Linear filtering matrix}

The linear filtering operation $S_{m} \in \mathbb{R}^{m^{2} \times n^{2}}$ is defined as

$$
S_{m}:=H \otimes H,
$$

where

$$
H:=T_{m}^{\prime}\left[\begin{array}{lll}
0_{m \times \frac{n-m}{2}} & I_{m} & 0_{m \times \frac{n-m}{2}}
\end{array}\right] T_{n} .
$$

\section{Appendix C. Direct transcription of (12) into a nonlinear program}

To numerically approximate the solution to the dynamic optimization problem (12), we transcribe it into a nonlinear program. To do this, we approximate the state and control trajectories by their values at $N_{c}$ times equispaced across the domain $[0, T]$. The $i^{\text {th }}$ time point is written $\tau_{i}:=\frac{T i}{N_{c}-1}, i=0, \ldots, N_{c}-1$, and so there are $N_{c}-1$ intervals of width $T_{u}:=\frac{T}{N_{c}-1}$. The vector $\tilde{X}^{i} \in \mathbb{R}^{m^{2}}$ approximates the state vector $\tilde{X}$ at time $\tau_{i}$, i.e. $\tilde{X}^{i} \approx \tilde{X}\left(\tau_{i}\right), i=0, \ldots, N_{c}-1$ and $u^{i} \in[-1,1]$ approximates the input at time $\tau_{i}$, i.e. $u^{i} \approx u\left(\tau_{i}\right)$. We then use a trapezoidal approximation [34] to write the state dynamics (12c) as an equality constraint in terms of $\tilde{X}^{i}, u^{i}$, $i=0, \ldots, N_{c}-1$ as

$$
\frac{\tilde{X}^{i+1}-\tilde{X}^{i}}{T_{u}}=\frac{1}{2}\left(\left(\alpha \tilde{A}_{\kappa}+\tilde{N}_{0}+u^{i+1} \tilde{N}\right) \tilde{X}^{i+1}+\left(\alpha \tilde{A}_{\kappa}+\tilde{N}_{0}+u^{i} \tilde{N}\right) \tilde{X}^{i}\right), \quad i=0, \ldots, N_{c}-2,
$$


where $\tilde{X}^{0}=\tilde{X}_{0}=\left[I_{m^{2}-1} 0_{\left(m^{2}-1\right) \times 1}\right] S_{m} X\left(j T_{r}\right)$. By defining $\mathrm{X} \in \mathbb{R}^{\left(m^{2}-1\right) \times N_{c}}, \mathrm{U} \in \mathbb{R}^{N_{c} \times 1}$ as

$$
\mathbf{X}:=\left[\begin{array}{c}
\left(\tilde{X}^{0}\right)^{\prime} \\
\left(\tilde{X}^{1}\right)^{\prime} \\
\vdots \\
\left(\tilde{X}^{N_{c}-1}\right)^{\prime}
\end{array}\right], \quad \mathbf{U}:=\left[\begin{array}{c}
u^{0} \\
u^{1} \\
\vdots \\
u^{N_{c}-1}
\end{array}\right]
$$

the dynamics (12c) can be written as a vector-valued constraint linear in $\mathrm{X}$ and linear in $\mathrm{U}$ but not jointly linear in both, thus:

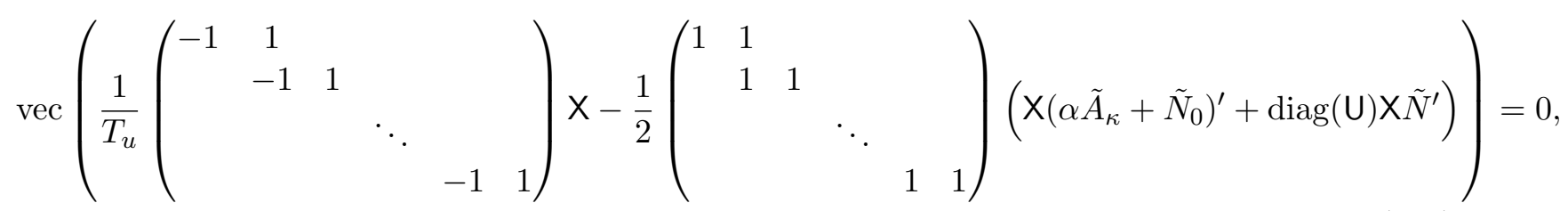

where vec denotes the linear, vectorize operation, $\operatorname{vec}\left(\begin{array}{ll}a & b \\ c & d\end{array}\right)=\left[\begin{array}{llll}a & c & b & d\end{array}\right]^{\prime}$.

In a similar fashion, the cost (12a) can be approximated in terms of $\tilde{X}^{i}, u^{i}, i=0, \ldots, N_{c}-1$ by a trapezoidal integration written as

$\int_{0}^{\infty} \frac{\beta}{2} \tilde{X}^{\prime}(\tau) \tilde{Q} \tilde{X}(\tau)+\frac{1}{2} r u^{2}(\tau) \mathrm{d} \tau \approx \sum_{i=0}^{N_{c}-2} \frac{\frac{\beta}{2}\left(\tilde{X}^{i+1}\right)^{\prime} \tilde{Q} X^{i+1}+\frac{\beta}{2}\left(\tilde{X}^{i}\right)^{\prime} \tilde{Q} X^{i}}{2} T_{u}+\frac{\frac{1}{2} r\left(u^{i+1}\right)^{2}+\frac{1}{2} r\left(u^{i}\right)^{2}}{2} T_{u}$.

Again this can be written directly in terms of $\mathrm{X}, \mathrm{U}$ thus:

$$
\mathrm{J}(\mathrm{X}, \mathrm{U}):=\frac{1}{4} T_{u}\left(\beta \operatorname{vec}\left(\mathrm{X}^{\prime}\right)^{\prime}\left(\left(\begin{array}{ccc}
1 & & \\
& 2 I_{N_{c}-2} & \\
& & 1
\end{array}\right) \otimes \tilde{Q}\right) \operatorname{vec}\left(\mathrm{X}^{\prime}\right)+r \mathrm{U}^{\prime}\left(\begin{array}{ccc}
1 & & \\
& 2 I_{N_{c}-2} & \\
& & 1
\end{array}\right) \mathrm{U}\right) .
$$

With the additional constraints on the input, the dynamic optimization problem (12) can therefore be approximated by

$$
\left(\mathrm{X}^{*}\left(X\left(j T_{r}\right)\right), \mathrm{U}^{*}\left(X\left(j T_{r}\right)\right)\right):=\arg \min _{\mathrm{X}, \mathrm{U}} \mathrm{J}(\mathrm{X}, \mathrm{U}),
$$

subject to (C.1), $\mathrm{X}^{\prime} e_{1}=\tilde{X}_{0}=\left[I_{m^{2}-1} 0_{\left(m^{2}-1\right) \times 1}\right] S_{m} X(0)$ where $e_{1}$ is the first canonical basis vector and $-\mathbf{1}_{N_{c}} \leq \mathrm{U} \leq \mathbf{1}_{N_{c}}$.

The trapezoidal approximation assumes a linear interpolation of the input inbetween collocation points [35]. The approximation of the optimal control law computed with this method is then written as

$$
u^{*}\left(X\left(j T_{r}\right), t\right) \approx \mathrm{U}_{\left\lfloor\frac{t}{T_{u}}\right\rfloor+1}^{*}+\frac{\mathrm{U}_{\left\lfloor\frac{t}{T_{u}}\right\rfloor+2}^{*}-\mathrm{U}_{\left\lfloor\frac{t}{T_{u}}\right\rfloor+1}^{*} t .}{T_{u}} t .
$$

To summarize, (C.2) is an optimization problem with $m^{2} N_{c}$ decision variables, $N_{c}$ equality constraints representing the state dynamics and initial condition and $2 N_{c}$ inequality constraints representing the saturating input. For more details on this direct transcription approach and alternatives, see [34, p. 89-92]. 


\section{References}

[1] I. Glasgow, N. Aubry, Enhancement of microfluidic mixing using time pulsing, Lab on a Chip 3 (2003) 114-120.

[2] E. L. Paul, V. Attiemo-Obeng, S. M. Kresta, Handbook of industrial mixing: science and practice, Wiley, 2003.

[3] J. M. Ottino, S. Wiggins, Introduction: mixing in microfluidics, Phil. Trans. R. Soc. Lond. A 362 (2004) $923-935$.

[4] F. J. Meacle, R. Lander, P. A. Shamalou, N. J. Titchener-Hooker, Impact of engineering flow conditions on plasmid DNA yield and purity in chemical cell lysis operations, Biotechnology and bioengineering 87 (2004) 293-302.

[5] H. Aref, Stirring by chaotic advection, Journal of Fluid Mechanics 143 (1984) 1-21.

[6] H. Stone, A. Stroock, A. Ajdari, Engineering flows in small devices: microfluidics toward a lab-on-a-chip, Annual review of fluid mechanics 36 (2004) 381-411.

[7] R. Griesse, K. Kunisch, Optimal control for a stationary MHD system in velocity-current formulation, SIAM J. Control and Optimization 45 (2007) 1822-1845.

[8] J.-Y. Dieulot, N. Petit, P. Rouchon, G. Delaplace, A torus model containing a sliding well-mixed zone as a way to represent mixing process at unsteady stirring conditions in agitated vessels, Chemical Engineering Communications 192 (2005) 805-826.

[9] J.-Y. Dieulot, N. Petit, P. Rouchon, G. Delaplace, An arrangement of ideal zones with shifting boundaries as a way to model mixing processes in unsteady stirring conditions in agitated vessels, Chemical Engineering Science 60 (2005) 5544-5554.

[10] O. M. Aamo, M. Krstic, T. R. Bewley, Control of mixing by boundary feedback in 2D channel flow, Automatica 39 (2003) 1597-1606.

[11] A. Balogh, O. M. Aamo, M. Krstic, Optimal mixing enhancement in 3D pipe flow, IEEE Transactions on Control Systems Technology 13 (2005) 27-41.

[12] D. D'Alessandro, M. Dahleh, I. Mezic, Control of mixing: A maximum entropy approach., IEEE Transactions on Automatic Control 44 (1999) 1852-1864.

[13] W. Liu, Mixing enhancement by optimal flow advection, SIAM Journal of Control and Optimization 47 (2008) 624-638.

[14] A. Vikhansky, Enhancement of laminar mixing by optimal control methods, Chem. Eng. Science 57 (2002) 2719-2725.

[15] G. Mathew, I. Mezic, S. Grivopoulos, U. Vaidya, L. Petzold, Optimal control of mixing in Stokes fluid flows, J. Fluid Mech. 580 (2007) 261-281.

[16] L. Cortelezzi, A. Adrover, M. Giona, Feasiblity, efficiency and transportability of short-horizon optimal mixing protocols, J. Fluid Mech. 597 (2008) 199-231.

[17] G. Mathew, I. Mezic, L. Petzold, A mutliscale measure for mixing and its applications, Physica D 3 (2005) $2314-2321$.

[18] E. P. Ryan, Optimal feedback control of bilinear systems, J. Optimization Theory and Applications 22 (1984) 333-362.

[19] A. Lozano, B. Yip, R. K. Hanson, Acetone: a tracer for concentration measurements in gaseous flows by planar laser-induced fluorescence, Experiments in Fluids 13 (1992) 369-376.

[20] J. Happel, H. Brenner, Low Reynolds number hydrodynamics with special applications to particulate media, Kluwer academic publishers group, 1983.

[21] C. Canuto, A. Quarteroni, M. Y. Hussaini, T. A. Zang, Spectral Methods in Fluid Dynamics, Springer, 1988.

[22] W. Liu, G. Haller, Strange eigenmodes and decay of variance in the mixing of diffusive tracers, Physica D 188 (2004) 1-39.

[23] M. Frigo, S. G. Johnson, The design and implementation of FFTW, Proceedings of the IEEE, Invited paper, Special Issue on Program Generation, Optimization, and Platform Adaptation 93 (2005) 216-231.

[24] S. P. Banks, Optimal control of distributed bilinear systems, Systems and Control Letters 9 (2) (1987) $183-189$.

[25] R. F. Curtain, A comparison of finite-dimensional controller design for distributed parameter systems, Control - Theory and Advanced Technology 9 (3) (1993) 609-628.

[26] L. N. Trefethen, Spectral Methods in MATLAB, SIAM, 2000.

[27] J. A. Weideman, S. C. Reddy, A MATLAB differentiation matrix suite, ACM Transactions on Mathematical Software TOMS 26 (2000) 465-519.

[28] J. P. Boyd, Chebyshev and Fourier spectral methods, Courier Dover Publications, 2001.

[29] S. Boyd, L. E. Ghaoui, E. Feron, V. Balakrishnan, Linear matrix inequalities in system and control theory, Vol. 10, SIAM Philadelphia, 1994.

[30] I. J. Couchman, E. C. Kerrigan, J. C. Vassilicos, Optimization-based feedback control of mixing in a Stokes fluid flow, in: Proc. European Control Conference, Budapest, Hungary, 2009, pp. 1227-1232. 
[31] J.-L. Thiffeault, G. A. Pavliotis, Optimizing the source distribution in fluid mixing, Physica D 237 (2008) 918-929.

[32] A. C. Hindmarsh, P. N. Brown, K. E. Grant, S. L. Lee, R. Serban, D. E. Shumaker, C. S. Woodward, SUNDIALS: Suite of nonlinear and differential/algebraic equation solvers, ACM Transactions on Mathematical Software 31 (2005) 363-396.

[33] A. Wächter, L. T. Biegler, On the implementation of a primal-dual interior point filter line search algorithm for large-scale nonlinear programming, Mathematical Programming 106 (2006) 25-57.

[34] J. T. Betts, Practical methods for optimal control using nonlinear programming, SIAM, 2001.

[35] D. G. Hull, Conversion of optimal control problems into parameter optimization ones, Journal of Guidance, Control and Dynamics 20 (1) (1997) 57-60. 